\title{
Fuzzy c-means based plant segmentation with distance dependent threshold
}

\author{
Mads Dyrmann \\ mady@mmmi.sdu.dk
}

\begin{abstract}
An important element in weed control using machine vision is the ability to identify plant species based on shape. For this to be done, it is often necessary to segment the plants from the soil. This may cause problems, if the colour of a plant is not consistent, since plants are then at risk of being separated into several objects. This study presents a plant segmentation method based on fuzzy c-means and a distance transform. This segmentation method is compared with four other plant segmentation methods based on various parameters, including the ability to maintain the plants as whole, connected components. The method presented here is found to be better at preserving plants as connected objects, while keeping the false positive rate low compared to commonly used segmentations techniques.
\end{abstract}

\section{Introduction}

Rising environmental awareness is leading to an increased demand for products from environmentally sustainable agriculture. Therefore researchers are looking for ways to optimize weed control by either carrying out a targeted spraying or a mechanical weed control. Many weed control techniques require, firstly, the determination and location of the species of weeds. Previously, researchers have used images in which the plants are segmented from the background and then identified based on their shape. A good segmentation is therefore essential to be able to correctly determine the species. One problem with using colours to segment plants is that often the colour of the stem is different from the colour of the leaves. This problem causes plants to be separated into multiple parts as their stems are not segmented correctly. A good segmentation should therefore not only be evaluated on the number of correctly identified pixels, but also on the ability to keep plant elements connected.

One of the most commonly applied segmentation technique is Excessive green (ExG), which was developed by Woebbecke et al. 따]. Excessive green is a scaling of the green chromaticity, which makes green plants stand out from the soil. An addition to this method is the subtraction of excessive red (ExR). Excessive red is also a weighting of the chromaticities which ensures a highlighting of the soil [四]. By subtracting ExR from ExG (ExGR), the difference between plants and soil in images is increased. Both ExG and ExGR are fast to compute and both methods are therefore well suited for real-time operation in the field. However, both ExG and ExGR can often result in low accuracy segmentations [, छ].

\begin{tabular}{|l|l|ll}
\hline 1 et al. & compare grey histograms from different colour spaces and colour weights for
\end{tabular} plant segmentation and find that $\mathrm{a}^{*}$ from the CIE $\mathrm{L}^{*} \mathrm{a}^{*} \mathrm{~b}^{*}$ colour space and the following 
weighting $1 / 4(2 G-R-B)$ provides the best bimodal images for thresholding. Other colour weighting methods are NDVI, RVI and DVI []], which use the near-infrared spectrum to improve segmentation by utilizing the fact that plants are highly reflective in the near-infrared spectrum compared to the soil. Eaursen et al. $\mathbb{Q}$ propose a Bayesian classiner for plant seg mentation. This method is used to combine different colour features including red, green, blue, and near-infrared colour wells, their chromaticities, and NDVI, ExG, and ExR. The method is found to be better than all of the individual features alone. Noordam et al. [ए] propose a Fuzzy c-means based segmentation for fruits in images, which takes into account the spatial placement of pixels. This is achieved by averaging out the cluster relationship in a pixel neighbourhood. A similar approach is used by chuang et al. [t] for medical imaging Using the neighbouring pixels helps in segmenting flat regions with impulse noise, but the approach will not be suitable for segmenting plants with thin leaves and stems as soil pixels will often dominate the areas nearby stems and leaves.

This study aims to use a fuzzy c-means algorithm and a distant-dependent threshold to segment plants from soil in RGB images while preserving stem regions of plants thus ensuring that leaves from a plant are not separated from each other in the segmentation process.

\section{Data material}

The images used are randomly sampled from previous studies and cover a variety of cameras, plants, soil types and lighting. All images are RGB images. The cameras that have been used are: Samsung EK-GN120, Samsung NX1000, JAI AD080-GE and Canon PowerShot SD1000.

The images from the Canon Powershot SD1000 are from [ $\square, \square]$ and consist of 3,000,816 pixels. The images from the JAI AD080-GE are from [ $[$ ] and consist of 3,755,808 pixels, the images from Samsung EK-GN120 consist of 2,419,116 pixels and the images from Samsung NX1000 consist of 21,892,117 pixels. Samples from the four cameras are shown in Figure 1. All images have been segmented by hand in order to create a ground truth for the segmentation. The images from [曰] come with segmentation masks created using NIR imaging. These masks have not been used in this study. The images are acquired with different backgrounds and under different light conditions. The images from the Samsung EK-GN120 and Samsung NX1000 are acquired outdoors in sunlight without shading. The images from JAI AD080-GE [日] are acquired outdoors in artificial light and the images from the Canon

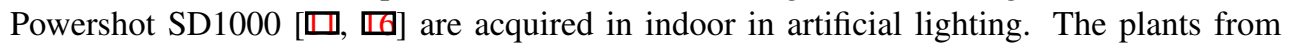
$[\square, \square]$ are planted in pots trays and photographed against a red plant tray. These images has been cropped in order to remove this red plant tray.

The images from the JAI AD080-GE [ 8 ] have a severe chromatic aberration, which results in green or purple colours in high-contrast areas and some of the images from the Canon Powershot SD1000 [ $\square, \square]$ have soil surface covered in green algae.

In total, 40 test images are used, which together contain 31,067,857 pixels, of which 4,199,568 are plant pixels.

\section{Methods}

In order to reduce the effects from different light conditions, the RGB images are converted to the CIE L*a*b* colour-space, whereby illumination can be neglected, by removing the 

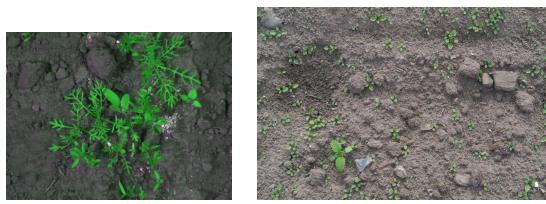

(a) JAI AD080-GE at (b) Samsung NX1000 $5 \%$ scale [日]
$1.5 \%$ scale

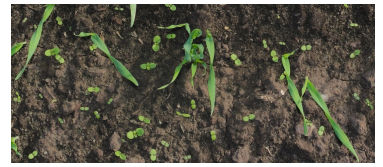

(c) Samsung EK-GN120 at 30\% scale

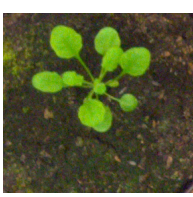

(d) Canon Powershot SD1000 at $80 \%$ scale [미, 미]

Figure 1: Samples from the four different cameras scaled for the same resolution

Luminosity channel. Because the images are acquired using different cameras and in different setups, no camera calibrations has been carried out as described in [ $\theta$ ]. The conversion from RGB to CIE L*a*b* is carried out using the CIE standard illuminant D65 [0], which simulates a daylight colour temperature of $6504 \mathrm{~K}$.

\subsection{Fuzzy $c$-means segmentation}

The fuzzy $c$-means algorithm is an unsupervised classification method in which pixels are given a membership to each $c$ cluster. After the membership assignment, the cluster centroids are updated based on pixel values and their membership assignments. This is done iteratively, until the positions of the cluster centroids settle.

Here two clusters are defined: a plant cluster and a soil cluster.

The image, consisting of $N$ pixels, is reshaped into a matrix, $\mathbf{X}_{N \times 2}$, with one row per pixel.

$$
\mathbf{X}_{N \times 2}=\left[\begin{array}{cc}
a_{1}^{*} & b_{1}^{*} \\
\vdots & \vdots \\
a_{N}^{*} & b_{N}^{*}
\end{array}\right]
$$

where $\mathrm{a}^{*}$ and $\mathrm{b}^{*}$ are the chromaticity indices from the CIE L*a*b* colour space. The centroid of cluster $\mathbf{c}_{i}$ is calculated as the weighted mean of the data $\mathbf{X}$ :

$$
\mathbf{c}_{i}=\frac{\sum_{n=1}^{N} u_{i n}^{\phi} \mathbf{x}_{n}}{\sum_{n=1}^{N} u_{i n}^{\phi}}
$$

where $u_{\text {in }}$ is the membership of the data-point $\mathbf{x}_{n}$ to cluster $\mathbf{c}_{i}$ and $\phi$ is the fuzzy exponent in the interval $\{\phi \in \mathbb{R} \mid 1<\phi\}$. In this study $\phi$ has been set to 2.1 , which empirically has shown to be a good value. The membership, $u_{i n}$, is the distance from $\mathbf{x}_{n}$ to class $i$ normalized by the sum of distances to both centroids:

$$
u_{i n}=\frac{1}{\sum_{j=1}^{2}\left(\frac{D_{i n}}{D_{j n}}\right)^{\frac{2}{\phi-1}}}
$$

$D_{\text {in }}$ is the intensity distance from pixel $n$ to centroid $i$. In this study, three distances have been tested: Euclidean, Mahalanobis, and Gustafson-Kessel [ $[\mathbf{Q}, \mathbf{0}]$, where the two latter 


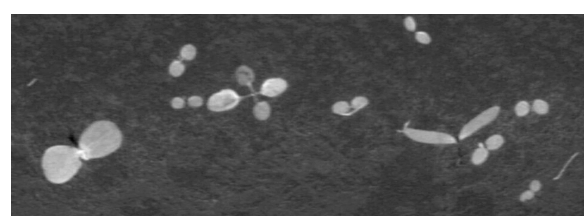

(a) Initial membership of plant class

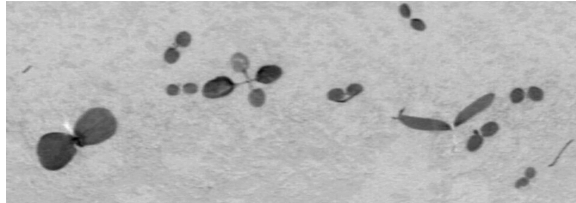

(b) Initial membership of soil class

Figure 2: Initial membership

allow non-spherical membership clusters. As a termination criteria, the difference in a cost function, $J$, between two iterations is used. $J$ is given by the sum of the distances from all data $\mathbf{x}_{n}$ to the cluster centres $\mathbf{c}_{i}$, multiplied with the corresponding degree of membership $u_{i n}$ of pixel $\mathbf{x}_{n}$ to cluster $\mathbf{c}_{i}$.

$$
J=\sum_{i=1}^{2} \sum_{n=1}^{N} \mathbf{u}_{i n}^{\phi} D_{i n}^{2}
$$

\subsection{Initialization}

The system can be initialized with no priors, which means that in the first iteration all pixels are assigned random membership of the two classes. Hereby, the system will find the two most distinct classes, but it will not provide information on which class is the plant class, and which one is the soil class.

A way to know which class is the plant class, while also lowering the number of iterations needed is to set the initial membership of the pixels. In this study the normalized difference between the $a^{*}$ and $b^{*}$ channel from the CIE $\mathrm{L} * \mathrm{a}^{*} \mathrm{~b} *$ image has been used as the prior membership.

$$
\mathbf{u}_{n \_ \text {init }}=\left[b_{n}^{*}-a_{n}^{*}, 1-\left(b_{n}^{*}-a_{n}^{*}\right)\right],
$$

where $a^{*}$ and $b^{*}$ are scaled to the interval $[0 ; 1]$. An example of this initial membership is shown in Figure 2. Another initialization, which will help to ensure a correct convergence, while also limiting the number of iterations needed, is to set the initial centroids of the clusters. By setting the initial value of one of the centroids to a green colour and the value of the other centroid to a light grey colour, the centroids will most certainly converge towards the plant and soil class respectively. Here $\operatorname{RGB}=[80,100,10]$ is used as the initial value for the plant centroid, and $\mathrm{RGB}=[80,70,60]$ is used as the initial value for the soil centroid.

\subsection{Problems with small plant coverage}

If the plants to soil ratio is small, the fuzzy c-means algorithm may fail to segment the plants from the soil. That is because even small differences in the soil can create clusters that are more distinct from each other than the plants are from the soil, based on the sum of distances. In order to make sure that the two clusters that are created consists of soil and plants, respectively, the dataset, $\mathbf{X}$, can be extended with artificial plant and soil pixels. By extending the dataset with the same number of plant and soil pixels, the plant to soil ratio will move towards $1 / 2$.

In order to ensure that these extra pixels are representative for the plant and soil clusters, 


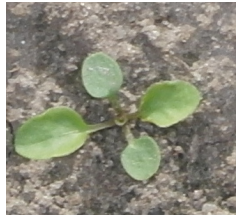

(a) input image

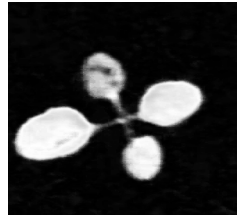

(b) membership plant class, $\mathbf{I}_{m}$

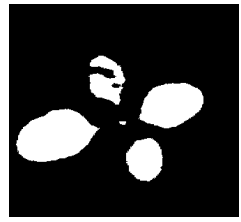

o (c) $B W_{\text {high }}$ created by (d) thresholding $\mathbf{I}_{m}$ at 0.8

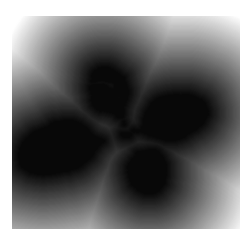

(d) $\mathbf{I}_{\text {dist }}$ created by (e) Final threshold making a distance made by thresholding transform of $B W_{\text {high }} \mathbf{I}_{m}$ at the value of the (contrast enhanced corresponding pixel for print) in $\mathbf{I}_{d} i s t$

Figure 3: input image and the assigned membership to the plant class

the extra pixels have to be updated for each iteration, so that they at all time correspond to the two cluster centroids. The drawback of extending the dataset is that more iterations are needed for the cluster convergence.

\subsection{Distance dependent threshold}

The class membership, $\mathbf{u}$, for all pixels, is used to create a grey-scale image, $\mathbf{I}_{m}$, in which pixels belonging to the plant class are close to 1, and pixels belonging to the soil class are close to 0 . Pixels that do not fit in neither the plant nor the soil classes will end up having values around 0.5 given that they are equally far from both classes. An example of such a membership image is shown in Figure 3(b). Pixels in the stem region of plants tend to have either a yellowish or a reddish colour, which means that they do not fit well in either the plant or the soil classes. Therefore, these pixels will be assigned membership values around 0.5. In order to make sure that these pixels are accepted as plant pixels, a variable threshold is made. This variable threshold depends on the distance to the nearest pixel with a high value. I.e. a pixel that most certainly is a plant pixel. This variable threshold ensures that pixels that have values around 0.5 , but which are far from plants, are not accepted as plant pixels, while the pixels that are located near by plants are accepted as plant pixels. In practice, the variable threshold is implemented by first thresholding $\mathbf{I}_{m}$ at 0.8

$$
B W_{\text {high }}=\mathbf{I}_{m}>0.8
$$

A threshold at 0.8 ensures that only pixels with a high membership to the plant class are kept. A distance transform image $\mathbf{I}_{\text {dist }}$ is created from $B W_{\text {high }}[\mathbb{}[\mathbf{\square}]$ in which pixels are assigned a value corresponding to the distance to the nearest pixel with the value 1 . $\mathbf{I}_{\text {dist }}$ is scaled to the interval [0.2:0.7] and used to determine the threshold level of $\mathbf{I}_{m}$; Values in $\mathbf{I}_{m}$ higher than $\mathbf{I}_{\text {dist }}$ are assigned a 1 and values lower that $\mathbf{I}_{\text {dist }}$ are assigned a 0 . Tests have shown that the scaling to the interval [0.2:0.7] provides a good segmentation of the plants. By lowering the lower limit, more false positives are added and by increasing the upper limit, more false negatives are added.

\section{Results}

In this section, the fuzzy c-means algorithm with three different distance measures is compared with common plant segmentation methods. These methods are excessive green $(\mathrm{ExG})[\square]$ 
thresholded with Otsu's method[ㅁ] ], excessive green - excessive red (ExGR)[四] thresholded with Otsu's method, difference between normalised $\mathrm{a}^{*}$ and normalised $\mathrm{b}^{*}$ thresholded with Otsu's method and Bayes segmentation[]].

The segmentation methods are compared using the following metrics:

Accuracy The number of pixels with a correct assignment relative to the total number of pixels. Higher is better.

Precision The number of pixels that are correctly assigned to plants relative to the total number of pixels assigned to plants. Higher is better.

Recall The number of pixels assigned to plants relative to the total number of plant pixels. Higher is better.

Mean distance to plant A measure of how close the edges of objects in the segmented image are to the plants in the hand-segmented image. This measure is created by finding the edges of the segmented image and for each pixel on the edge finding the distance to the nearest pixel on the edge of the hand-segmented image. Lower is better.

Variance of distance to plant This measure is the variance of the distances of all edge pixels in the segmented image to the nearest edge on a hand-segmented image. This measure explores how rough the edges are of the segmented image. If the variance is small, the segmented image will look like an eroded/dilated version of the hand-segmented image. Lower is better.

Mean object precision This is the precision object-by-object. This measure is only calculated for the objects in the segmented image, that touches plants in the hand-segmented image. A high value indicates that, for the plants that have been found, most of the pixels of the plans have been found. Higher is better.

Mean object recall The measure calculates the average recall object-by-object. This measure is only calculated for the objects in the segmented image that touch plants in the handsegmented image. A high object recall indicates that, for the detected plants, there are few false positives. Higher is better.

Fraction of objects split in multiple parts If a plant is split in multiple parts in the segmented image, this measure will increase. This measure only counts the number of plants that are split in multiple parts relative to the total number of plants. The measure does not take into account how many objects the plants are split into, how big the plants are nor how small the individual objects are. Lower is better.

Figure 4 shows of high and low accuracy, precision, recall and distance for four plants.

The results provided in table 1 are averaged over pixels or objects. E.g. the accuracy is calculated by summing the correct identified pixels for all images and divide by the total number of pixels in all images. As can be seen, all seven methods provide a high accuracy. The precision achieved using the three fuzzy-c means methods outperform the other methods. However, all methods except for Bayes provide a precision over 0.9. The recall 


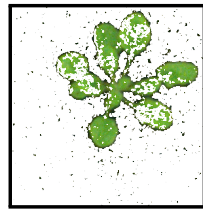

(a) low accuracy, pression, low recall

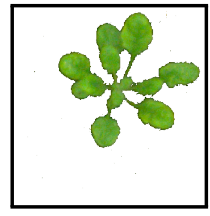

low (b) high accuracy, high (c) precission, high recall
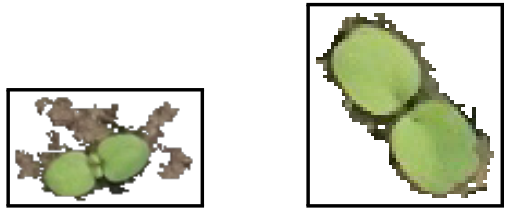

(c) high precission, low (d) medium mean disaccuracty, low recall, high tance, low dist variance dist variance

Figure 4: Examples on metrics for four different plants

Table 1: Results for the different classifier methods. The best performances are underlined.

\begin{tabular}{l|llllllll}
\hline & Accuracy & Precision & Recall & $\begin{array}{l}\text { Mean dis- } \\
\text { tance }\end{array}$ & $\begin{array}{l}\text { Var. of } \\
\text { distances }\end{array}$ & $\begin{array}{l}\text { Mean obj. } \\
\text { precision }\end{array}$ & $\begin{array}{l}\text { Mean obj. } \\
\text { recall }\end{array}$ & $\begin{array}{l}\text { Frac. of } \\
\text { split obj. }\end{array}$ \\
\hline Bayes & 0.945 & 0.745 & 0.677 & 29.9 & 3232.6 & 0.042 & $\underline{0.969}$ & 0.637 \\
ExG & 0.983 & 0.911 & 0.902 & 13.3 & 1005.5 & 0.246 & 0.884 & 0.404 \\
ExGR & 0.977 & 0.927 & 0.832 & 14.1 & 1078.6 & 0.294 & 0.852 & 0.340 \\
$\mathrm{a}^{*}-\mathrm{b}^{*}$ & $\underline{0.989}$ & 0.951 & $\underline{0.924}$ & $\underline{4.0}$ & $\underline{282.4}$ & 0.385 & 0.957 & 0.442 \\
fcm $_{\text {Euclid }}$ & 0.988 & 0.978 & 0.897 & 6.7 & 482.2 & 0.769 & 0.884 & 0.151 \\
fcm $_{\text {GK }}$ & 0.968 & $\underline{0.990}$ & 0.740 & 8.4 & 563.8 & 0.862 & 0.799 & 0.083 \\
fcm $_{\text {Mahal }}$ & 0.953 & $\underline{0.990}$ & 0.658 & 11.4 & 689.3 & $\underline{0.889}$ & 0.706 & $\underline{0.068}$ \\
\hline
\end{tabular}

of $a^{*}-b^{*}$ is the highest at 0.92 , which means that the number of true positives is close to the number of plant pixels. The mean distance and variance of distances for $\mathrm{a}^{*}-\mathrm{b}^{*}$ are the lowest of the seven methods, indicating that the edges of the segmentation are closest to the real edges of the plants. The fuzzy c-means methods are slightly worse, though still better than ExG, ExGR and Bayes. On the individual objects, the recall of Bayes method is the best. It has, however, a very low overall recall indicating that for some objects only few of the total number of plant pixels have been found. The number of plants that has been split in multiple parts is the lowest for the three fuzzy c-means based methods.

Figure 5 shows a composed image of plants segmented using the seven methods. This example shows how the fuzzy c-means methods are able to detect some stems even though they are non-green. The edges of the objects are, however, often a few pixels off relative to the real edge of the leaves, which is partly due to the distance-dependent threshold. As can be seen in Figure 5(h) the edges of fcm Mahal are rough compared to ExG and ExGR even though the mean distance is lower. That is because the segmentation from ExG and ExGR creates more false objects far from the edges of the plants, which thus increase the mean distances of the edges.

\subsection{Sensitivity towards algae and uneven backgrounds}

Some of the 40 test images contain algae or gravel backgrounds with big variation. Since these images account for only a few of the 40 images, they have little effect on the statistics. However, when choosing the right segmentation method, the robustness toward algae should be taken into account. For the images with algae on the soil all algorithms have problems. Such a case is shown in Figure 6. 


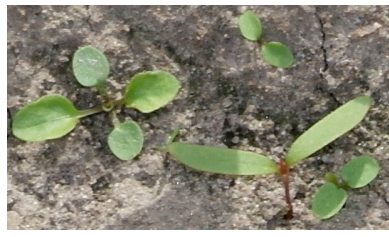

(a) RGB input

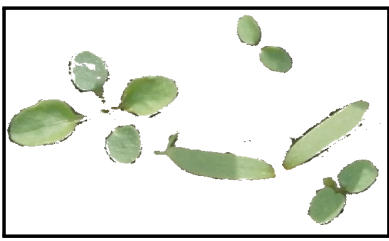

(d) Segmentation using ExG-ExR

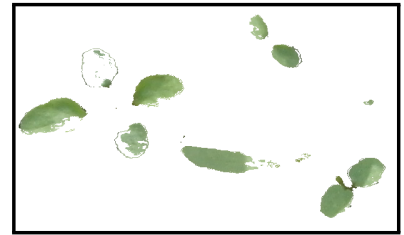

(b) Segmentation using naive Bayes approach

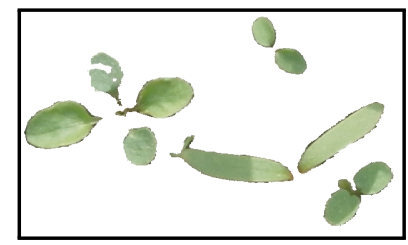

(e) Segmentation using $a^{*}-b^{*}$

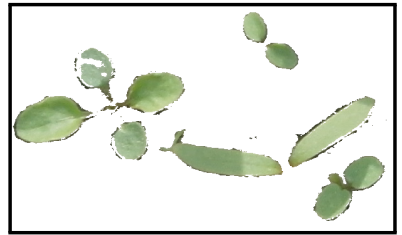

(c) Segmentation using ExG

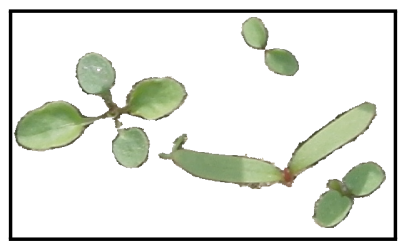

(f) Segmentation using fuzzy cmeans with euclidean distance

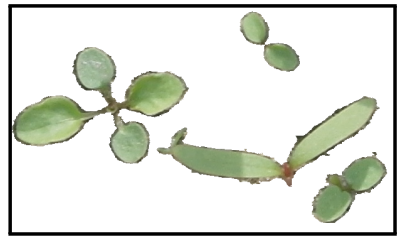

(g) Segmentation using fuzzy cmeans with Mahalanobis distance

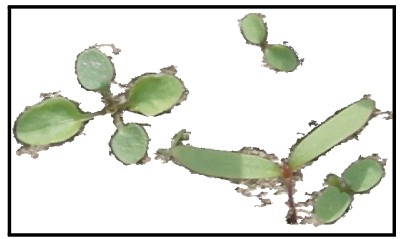

(h) Segmentation using fuzzy cmeans with Gustafson-Kessel distance

Figure 5: Plants segmented using seven different segmentation methods.(b) The segmentation using Bayes classifier has only few false plant elements, but it misses big parts of plants. (c)(d) The segmentation using ExG and ExGR captures most of the leaves but misses some stems. (e) The segmentation using $a^{*}-b^{*}$ captures most of the leaves and provides the sharpest edges, but it misses some stems. (f)(g)(h) The fuzzy c-means methods are all able to detect the stems. The edges, however, is not as sharp as $\mathrm{a}^{*}-\mathrm{b}^{*}$.

\section{Discussion}

Previously segmentation algorithms in the plant domain have mainly been evaluated on their accuracy, but the ability to preserve shape might in some cases be more relevant. The shape, however, is changed greatly if the leaves of the plant are separated from the stem in the segmentation process.

All seven segmentation methods provide a high accuracy, which should be seen in the light of the plant to soil ratio, which is $15.6 \%$. Of the seven segmentation methods, the $\mathrm{a}^{*}-\mathrm{b}^{*}$ method gives the best accuracy, recall, mean segmentation distance and distance variance. The methods is, however, not as good at keeping leaves connected as the fcm-based methods. Therefore, based on this study, the $\mathrm{a}^{*}-\mathrm{b}^{*}$ method is the best methods for e.g. determine the amount of biomass in an image. It may, however, not be the best choice for a shape based classification.

Because of the high recall and precision, the Euclidean distance is the best for the fuzzy c-means algorithm as it most often results in plants that are connected, while the false posi- 


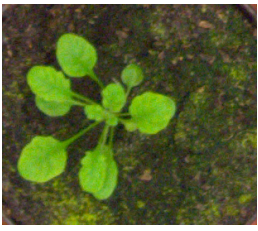

(a) RGB input

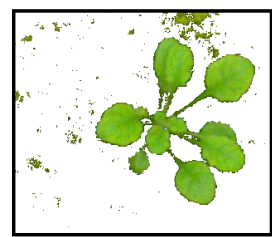

(e) $a^{*}-b^{*}$

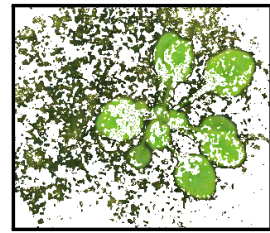

(b) Bayes

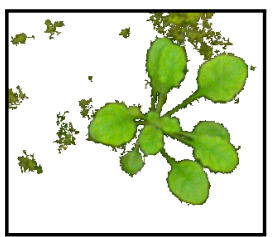

(f) $\mathrm{fcm}_{\text {Euclid }}$

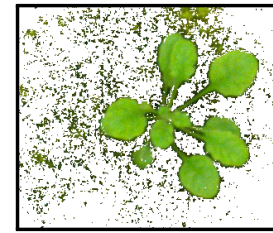

(c) $\mathrm{ExG}$

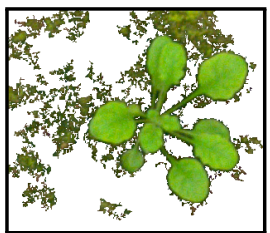

(g) $\mathrm{fcm}_{\mathrm{GK}}$

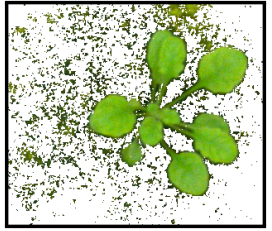

(d) ExGR

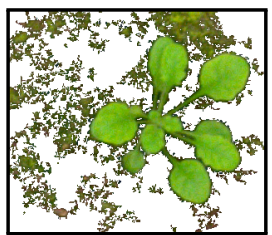

(h) fcm Mahal

Figure 6: example of image with algae where all algorithms fail at removing the background. However, Some of these results could be improved by simple post-filtering.

tives rate is kept low. Compared to ExG and ExGR, the fcm-segmentation with a Euclidean distance decreases the number of plants split in multiple parts by $62 \%$ and $56 \%$ respectively. Another benefit of the fcm-based segmentation methods is their ability to handle non-green plant elements such as red stems. This ability is caused by the fact that red stems fit poorly in both the plant and soil class, whereby they can be found by using a distance-dependent threshold. This distance-dependent threshold, however, has the drawback that the edges of the segmented plants are not as smooth as e.g. the edges on plants segmented using $\mathrm{a}^{*}-\mathrm{b}^{*}$. The Bayesian classifier used in this comparison has been trained with RGB images acquired by different cameras in different fields and under different light conditions. The results achieved in this study are therefore not directly comparable with the results obtained by caursen et al.|ष], who use RGB+NIR images acquired using only one camera in one held.

None of the algorithms have been measured on how computationally expensive they are. However, the implementation of the fuzzy c-means algorithms used in this study is not suitable for real-time operation in the field, as it takes several seconds to process each image on a $2.6 \mathrm{GHz}$ Intel i5 processor.

\section{Conclusion}

Segmentation of plants with fuzzy c-means has proven to be a viable alternative to established segmentation methods. The method has been compared and evaluated on various parameters including the ability to preserve objects connected. The edges of objects segmented by the fuzzy c-means methods are closer to the hand-segmented objects than objects segmented using ExG, ExGR and Bayesian segmentation. However the $\mathrm{a}^{*}-\mathrm{b}^{*}$ methods results in objects with the edges closest to the hand-segmented object. For object classification based on shape, the fuzzy c-means segmentation method is the best of the tested methods in terms of preserving the shape of plants. 


\section{References}

[1] Xiaodong Bai, Zhiguo Cao, Yu Wang, Zhenghong Yu, Zhu Hu, Xuefen Zhang, and Cuina Li. Vegetation segmentation robust to illumination variations based on clustering and morphology modelling. Biosystems Engineering, 125:80-97, 2014. ISSN 15375110. doi: 10.1016/j.biosystemseng.2014.06.015. URL http://dx.doi . org/10.1016/j.biosystemseng.2014.06.015.

[2] Keh-Shih Chuang, Hong-Long Tzeng, Sharon Chen, Jay Wu, and Tzong-Jer Chen. Fuzzy c-means clustering with spatial information for image segmentation. Computerized medical imaging and graphics : the official journal of the Computerized Medical Imaging Society, 30(1):9-15, 2006. ISSN 0895-6111. doi: 10.1016/j.compmedimag. 2005.10.001.

[3] Mads Dyrmann and Peter Christiansen. Automated Classification of Seedlings Using Computer Vision. Technical report, Aarhus University, Aarhus, 2014. URL http://plant_recognition.sdu.dk/files/ AutomatedClassificationofseedlingsUsingComputerVision.pdf.

[4] Ashish Gupta and Richard Bowden. Fuzzy encoding for image classification using Gustafson-Kessel algorithm. Proceedings - International Conference on Image Processing, ICIP, pages 3137-3140, 2012. ISSN 15224880. doi: 10.1109/ICIP.2012. 6467565 .

[5] Sebastian Haug and Jörn Ostermann. A Crop/Weed Field Image Dataset for the Evaluation of Computer Vision Based Precision Agriculture Tasks. In Computer Vision - ECCV 2014 Workshops, volume 8928, pages 105-116. Zurich, Switzerland, 2015. ISBN 9783319162201. doi: 10.1007/978-3-319-16220.

[6] Ronghua Ji, Zetian Fu, and Lijun Qi. Real-time plant image segmentation algorithm under natural outdoor light conditions. New Zealand Journal of Agricultural Research, 50 (January 2015):847-854, 2007. ISSN 0028-8233. doi: 10.1080/00288230709510359.

[7] Deane B. Judd, David L. Macadam, Günter Wyszecki, H. W. Budde, H. R. Condit, S. T. Henderson, and J. L. Simonds. Spectral Distribution of Typical Daylight as a Function of Correlated Color Temperature. Journal of the Optical Society of America, 54(8): 1031, 1964. ISSN 0030-3941. doi: 10.1364/JOSA.54.001031.

[8] Morten Stigaard Laursen, Henrik Skov Midtiby, Norbert Krüger, and Rasmus Nyholm Jørgensen. Statistics-based segmentation using a continuous-scale naive Bayes approach. Computers and Electronics in Agriculture, 109:271-277, November 2014. ISSN 01681699. doi: 10.1016/j.compag.2014.10.009. URL http:// linkinghub.elsevier.com/retrieve/pii/s0168169914002567.

[9] Katherine León, Domingo Mery, Franco Pedreschi, and Jorge León. Color measurement in $\mathrm{L}^{*} \mathrm{a} * \mathrm{~b} *$ units from RGB digital images. Food Research International, 39(10): 1084-1091, 2006. ISSN 09639969. doi: 10.1016/j.foodres.2006.03.006.

[10] George E. Meyer and João Camargo Neto. Verification of color vegetation indices for automated crop imaging applications. Computers and Electronics in Agriculture, 63(2):282-293, October 2008. ISSN 01681699. doi: 10.1016/j.compag. 
2008.03.009. URL http://linkinghub.elsevier.com/retrieve/pii/ s0168169908001063.

[11] Massimo Minervini, Mohammed M. Abdelsamea, and Sotirios a. Tsaftaris. Imagebased plant phenotyping with incremental learning and active contours. Ecological Informatics, (available online 6 August 2013). ISSN 15749541. doi: 10.1016/j.ecoinf. 2013.07.004.

[12] J.C. Noordam, W.H.a.M. Van Den Broek, and L.M.C. Buydens. Geometrically guided fuzzy C-means clustering for multivariate image segmentation. Proceedings 15th International Conference on Pattern Recognition. ICPR-2000, 1(FEBRUARY 2000), 2000. ISSN 1051-4651. doi: 10.1109/ICPR.2000.905376.

[13] N Otsu. A Threshold Selection Method from Gray-Level Histograms. IEEE Transactions on Systems, Man, and Cybernetics, 9(1):62-66, 1979. ISSN 0018-9472. doi: 10.1109/TSMC.1979.4310076. URL http://ieeexplore.ieee.org/ lpdocs / epic03/wrapper.htm?arnumber $=4310076$.

[14] Azriel Rosenfeld. Distance functions on Digital Pictures. Pattern Recognition, 1:3361, 1986. ISSN 01678655. doi: 10.1016/0167-8655(86)90017-6.

[15] Rouse, J. W., Haas, R. H., Scheel, J. A., Deering, D. W. Monitoring Vegetation Systems in the Great Plains with ERTS. Third ERTS Symposium Abstract, page 20, 1974. URL http://ntrs.nasa.gov/archive/nasa/casi.ntrs.nasa. gov/19740003048.pdf.

[16] Hanno Scharr, Massimo Minervini, Andreas Fischbach, and Sotirios A Tsaftaris. Annotated Image Datasets of Rosette Plants. Forschungszentrum Jülich, (Technical Report No. FZJ-2014-03837), 2014.

[17] David M. Woebbecke, George E. Meyer, K. Von Bargen, and D. A. Mortensen. Color Indices for Weed Identification Under Various Soil, Residue, and Lighting Conditions. Transactions of the ASAE, 38(1):259-269, 1995. ISSN 2151-0059. doi: 10.13031/ 2013.27838. URL http://elibrary . asabe.org/abstract.asp??JID= $3 \& \mathrm{AID}=27838 \& \mathrm{CID}=\mathrm{t} 1995 \& \mathrm{~V}=38 \& i=1 \& \mathrm{~T}=1$. 